Revista Signos 2008, 41(67) $231-255$

\title{
Cognición, metacognición y escritura
}

\author{
Nelsi Lacon de De Lucia \\ Susana Ortega de Hocevar \\ Universidad Nacional de Cuyo \\ Argentina
}

Resumen: Este artículo presenta una contribución a la compleja problemática de la enseñanza de la escritura. Tiene como base tanto investigaciones teóricas como empíricas realizadas con alumnos de escolaridad común de nivel básico y medio. En primer lugar, explicitamos el marco teórico. A continuación, enfatizando especialmente los aspectos cognitivos y metacognitivos, desarrollamos un modelo didáctico de fases interactivas para la enseñanza de la producción escrita, el cual fue aplicado en cursos experimentales. Finalmente, exponemos las principales conclusiones.

Palabras Clave: Competencia, escritura, cognición, metacognición.

Recibido: 14-VIII-2006 Aceptado: $11-I-2008$
Correspondencia: Nelsi Lacon de De Lucia (nelsidel@logos.uncu.edu.ar). Tel.: (00-54-0261) 4135004 Fax: 4380457. Facultad de Filosofía y Letras, Universidad Nacional de Cuyo. Parque General San Martín, Ciudad (5500), Mendoza, Argentina. 


\section{Cognition, metacognition, and writing}

Abstract: This article presents a contribution to the complex problems of teaching how to write. It is based both on theoretical and empirical researche conducted on basic and medium level regular schooling students. First, the theoretical framework is explicitly stated. Then, the cognitive and metacognitive aspects are emphasizing a didactic model of interactive phases for the teaching of written production is developed, which was applied in experimental groups of student. Finally, the main conclusions are presented.

Key Words: Competence, writing, cognition, metacognition.

\section{INTRODUCCIÓN}

En este artículo presentamos un modelo didáctico de producción de textos escritos surgido de investigaciones tanto teóricas como empíricas, realizadas en torno a la problemática de la enseñanza y la evaluación de la producción de textos escritos ${ }^{1}$. El diseño experimental que elaboramos y pusimos en práctica se apoya fundamentalmente en modelos cognitivos, sociocognitivos y psicolingüísticos. Asimismo, en los aportes de investigadores que señalan la importancia de la metacognición en los aprendizajes. Además, sumamos a estas bases teóricas, el producto de nuestras reflexiones y experiencias en situaciones reales de clase.

Todo ello sirvió de fundamento a nuestras decisiones sobre el qué enseñar y cómo enseñar, sobre qué aspectos son claves en la producción escrita y cuáles son las estrategias didácticas más eficaces para desarrollar la competencia productiva de los alumnos. En función de tales propósitos diseñamos un modelo didáctico en el que se conjugan estrategias de tipo cognitivo, lingüístico y metacognitivo. El modelo tiene como principal objetivo que los alumnos desarrollen su competencia productiva. Ello implica que construyan una representación adecuada de la tarea de escritura en la memoria, lo que supone el conocimiento de estrategias de producción y su uso consciente por parte de los aprendices como así también la reflexión sobre la necesidad y utilidad de los procedimientos estratégicos.

Si bien en nuestras investigaciones hemos trabajado la producción de textos expositivo-descriptivos (nivel escolar medio) y narrativos (nivel escolar básico), consideramos que el procedimiento aplicado podría ser tenido en cuenta en la enseñanza sistemática de la producción escrita de distintos tipos de textos. En estos casos, habría que atender a las características propias de los mismos, en la planificación de tareas específicas.

En este artículo mostramos el modelo experimental aplicado a la enseñanza de la producción de textos expositivo-descriptivos, puesto en práctica con alumnos del nivel medio $-9^{\circ}$ de Educación General Básica, tercer ciclo (EGB3)- de una escuela de escolaridad común de la ciudad de Mendoza, República Argentina. La metodología empleada para concretar la investigación fue de tipo descriptivo, experimental y longitudinal debido a que se siguieron dos grupos -grupo A, de estudio y grupo B, de control- dentro de un determinado período. El Grupo A recibió el apoyo 
metodológico según lo explicitado en nuestro modelo, mientras que el grupo de control no participó de nuestro entrenamiento especial en estrategias de escritura.

Dos son los corpus que sirven de base para este estudio y que hemos relacionado para la obtención de conclusiones. Uno, constituido por textos expositivo-descriptivos producidos por los adolescentes en dos instancias: la primera, de diagnóstico -pretest- como evaluación inicial exploratoria de las habilidades de producción de estos estudiantes y la segunda, de evaluación final -postest-. El otro corpus, conformado por los informes metacognitivos escritos por los estudiantes luego de cada una de las instancias de producción.

\section{La escritura}

\subsection{La competencia productiva}

Dado que la producción de textos y los modelos que se ocupan de la misma presuponen que los sujetos deben desarrollar una determinada competencia, iniciamos nuestro trabajo precisando el concepto de competencia "un saber hacer que, de modo espiralado, se va construyendo a lo largo de la vida y comprende diversos aspectos de la acción humana" (Gómez de Erice, 2003: 23). Esta competencia no se adquiere por simple experiencia de vida sino que su desarrollo exige un trabajo pedagógico formal y sistemático.

Adoptamos también el concepto de competencia comunicativa de Hymes (1962), entendida como la capacidad que posee el ser humano de dominar distintas situaciones de habla y de escritura y emplear adecuadamente diferentes géneros y tipos de textos apropiados para las múltiples situaciones comunicativas con que se enfrenta en las prácticas sociales.

Asimismo, sumamos a este concepto la apertura de la competencia comunicativa en tres grandes competencias, realizada por Badura (1972). Para este investigador la competencia comunicativa está conformada por tres competencias: la lingüística, la hermenéutico-analítica (comprensiva) y la táctico-retórica (productiva). Si bien en todo acto de comunicación el empleo de estas tres subcompetencias es simultáneo nos referiremos específicamente a la táctico-retórica, ya que es la que posibilita al sujeto conocer e internalizar las estrategias discursivas necesarias para la elaboración de textos efectivos y adecuados a las distintas situaciones comunicativas.

La competencia táctico-retórica está constituida por dos componentes:

1. Textual: la capacidad de estructurar textos coherentes y cohesionados a nivel de superficie. Este componente, indispensable tanto en la comprensión como en la producción, facilita los procesos de metacognición en el acto de escritura. Cuando el sujeto advierte que algo no está bien en su texto puede pasar de la función epilingüística a la metalingüística y metadis- 
cursiva, es decir, identificar el origen de su error y ubicarlo ya sea en el plano de la norma lingüística o en el de la organización del discurso.

2. Estratégico-pragmático: la capacidad de elaborar discursos que respondan a las necesidades pragmáticas propias de cada práctica social. Para ello se requiere un conocimiento que permita identificar la práctica social a la que pertenecen los textos, como así también las reglas que los configuran.

\section{2. ¿Qué entendemos por escribir?}

Dado que escribir es un acto complejo, no es fácil responder esta interrogante de una manera breve y simple. Al respecto, coincidimos con Hayes (1996: 2), quien considera que escribir "es un acto comunicativo que requiere de contexto social y de un medio. Es una actividad generativa que requiere motivación, y una actitud intelectual que exige procesos cognitivos y memoria".

Adoptar esta conceptualización de Hayes, que es sustentada por los postulados epistemológicos sociocognitivos, en los que se enfatizan los aspectos sociales, culturales, motivacionales, afectivos y cognitivos de la escritura, nos llevó a centrarnos básicamente en los modelos cognitivos y sociocognitivos de producción escrita y en las estrategias implicadas en los mismos, como asimismo en las reflexiones sobre la incidencia de la metacognición en la escritura. La inclusión de modelos cognitivos y aportes sociocognitivos (Flower \& Hayes, 1980, 1981, 1996; Bereiter \& Scardamalia, 1987; Camps, 1993, 1997; Camps \& Castelló, 1996; Hayes, 1996; Castelló, 2003) y psicolingüísticos (van Dijk \& Kintsch, 1983), permite que se comprendan tanto los procesos que realiza la mente al producir un texto, como la incidencia de la situación comunicativa, la motivación, las creencias y predisposiciones del escritor que se evidencian en el texto escrito. Además, el modelo psicolingüístico de van Dijk y Kintsch (1983), centrado en diversos niveles estratégicos a partir de un texto, aporta un andamiaje teórico preciso a la hora de diseñar criterios y pautas de escritura y de evaluación del producto obtenido. Los autores de los modelos que hemos analizado coinciden en señalar que el aumento de la conciencia -estrategias metacognitivas- sobre los propios procesos de planificación, textualización y revisión favorece el desarrollo de la competencia productiva y permite, en consecuencia, el logro de un escritor eficiente.

Estas consideraciones están presentes ya en los primeros modelos cognitivos. Al respecto, Flower y Hayes (1981) señalan la necesidad de acrecentar el control sobre el propio proceso de composición para llegar a ser un escritor más eficiente y más efectivo con sus lectores.

En la actualidad los estudios acerca de la incidencia de la metacognición en la competencia productiva se han acentuado. Parodi (2003: 119) afirma:

"La capacidad metacognitiva del sujeto se contempla como un componente central en el desarrollo de un buen escritor. Sin un desarrollo adecuado de ella, el 
sujeto no logra ejercer el dominio de la situación de escritura y no alcanza a visualizar el problema retórico por resolver. Solo si se da cuenta y está consciente de que debe llevar a cabo una determinada tarea y de los recursos disponibles, podrá dimensionar su problema y buscar y ejecutar estrategias tendientes a una solución pertinente".

En síntesis, las bases teóricas mencionadas permiten conformar una concepción de la escritura fundada en tres aspectos esenciales que desarrollamos a continuación.

\subsubsection{La escritura: una actividad social y cultural}

Desde el punto de vista de la enseñanza de la lengua escrita, nos interesa destacar, en este marco, algunos aspectos que hay que considerar en el proceso de escritura. Al respecto, sintetizamos lo señalado por Camps (1997):

a) El lenguaje escrito está destinado a lectores que no comparten ni el tiempo ni el lugar del contexto de la producción. Debido a ello, el escritor debe realizar lo que Vygotsky (1979) denomina un proceso de "descontextualización" de este contexto, para crear un nuevo contexto. Para lograrlo, el lenguaje debe ser explícito. Esta transformación surge por una exigencia social del empleo de lenguaje escrito en una sociedad alfabetizada, en la que los intercambios comunicativos con los demás son tanto orales como escritos.

b) Desde la perspectiva de Bajtín (1982), además, es social por su naturaleza interactiva, "dialógica". En este sentido afirma que el lenguaje escrito es dialógico por dos motivos: 1. La mayor parte de los textos o enunciados no se constituyen en un primer texto o en un texto independiente de los demás sino que están insertos en un entramado comunicativo que hace posible su interpretación. El autor "dialoga" con lo que otros han dicho e imagina las respuestas de sus posibles lectores que anticipa en su posible texto. 2. De ello se infiere que el escritor no está en soledad creando su texto sino que está en un diálogo constante con distintas voces que surgen del contexto de esa situación comunicativa. El autor hace hablar en su texto a estas voces por lo que los textos son polifónicos.

Estas consideraciones nos llevan a reflexionar acerca de la importancia que tiene el conocimiento del destinatario, de los distintos contextos y del uso de la lengua en estos contextos, de los conocimientos y presuposiciones que se comparten, entre otros aspectos, para que la comunicación escrita sea exitosa.

Por ello, priorizamos en el modelo diseñado para la enseñanza de la producción textual el co- 
nocimiento de los usos adecuados de la lengua en cada situación comunicativa específica, que requiere formas propias de lenguaje, subordinadas o dependientes de ese contexto de uso.

\subsubsection{Una actividad que involucra lo afectivo y lo motivacional}

Los aspectos afectivos y motivacionales de la escritura están estrechamente vinculados a lo considerado en el apartado anterior. De hecho, cuando un escritor produce su texto, en tanto actividad social, insertándolo en un entorno socio-cultural determinado, es evidente que lo hace porque está consciente de los valores que tiene para él y para sus lectores esta actividad.

Las motivaciones de los "nuevos escritores" o "aprendices" están relacionadas con la función que le atribuyan al texto en una situación comunicativa determinada. De ello se desprende la necesidad, en la enseñanza de la producción textual, de escribir para situaciones comunicativas auténticas, que se constituyan en una motivación real para el alumno. Somos conscientes de que esto no siempre es fácil de conseguir, por ello nos interesa destacar -en tanto solución simple y eficaz para esta problemática- lo propuesto por Dolz (1994), quien tipifica tres situaciones de comunicación que originan actividades de escritura en la escuela, estas son:

- Auténticas, es decir, contextos extraescolares verdaderos, por ejemplo, cartas, cartas al lector, solicitudes, anuncios, afiches, folletos, encuestas, etc.

- Discursos del ámbito escolar, esto es, situaciones auténticas dentro del ámbito escolar, tales como diario o revista escolar, murales, folletos, afiches, etc.

- Ficcionalización, es decir, contextos inventados que mantienen similitud con la realidad, por ejemplo, simulaciones (simular ser un ecologista y escribir a favor de... o un abogado y argumentar a favor o en contra de......), juegos de rol, etc.

Otra consideración a tener en cuenta es que no todos los grupos sociales otorgan la misma valoración a la lengua escrita; ello también tiene gran incidencia en el aprendizaje. Al respecto, se han realizado numerosas investigaciones y todas coinciden en señalar que la escuela debe también ocuparse en trabajar este aspecto en forma sistemática a fin de lograr que la comunidad en la que está inserta valorice el uso de la escritura.

\subsubsection{La escritura: una actividad cognitiva compleja}

Este aspecto es el que subyace a todos los modelos cognitivos. Al respecto, Salvador Mata (1997: 26) destaca:

- "La escritura supone procesos y actividades cognitivas que, a su vez, implican varios subprocesos organizados en un sistema jerárquico, en cuyo nivel más alto se sitúa el control del proceso global. 
- La composición es un proceso que conduce a la integración de la información en el mismo nivel y entre distintos niveles, lo que supone un alto componente de regulación.

- La escritura tiene carácter flexible, recursivo e interactivo.

- Los procesos y la estructura de la composición están afectados y controlados por variables internas (conocimiento previo de restricciones lingüísticas y del tema de la escritura) y externas (contexto comunicativo y audiencia)".

En síntesis, de las consideraciones expuestas se desprenden dos premisas básicas que tuvimos en cuenta para la elaboración de nuestro modelo: la necesidad de pensar en un modelo educativo para la enseñanza de la producción escrita que aúne los aspectos sociales, cognitivos, afectivos y motivacionales. La inserción de la producción escrita en auténticos contextos comunicativos.

\section{Metacognición}

Siguiendo la línea de los modelos cognitivos interactivos que hemos explicitado, actualmente se concibe a la producción escrita como una actividad de construcción de significados que se estructura alrededor de un proceso cognitivo y que debe responder a condicionamientos de diversa índole. Para responder a estas demandas, los escritores y en un sentido más amplio, los hablantes, poseen conocimientos generales que han adquirido en su proceso de socialización los cuales guían su comprensión y producción textual. Según Heinemann y Vieweger (1991), estos saberes serían de distinto carácter: un saber enciclopédico referido al conocimiento del mundo, un saber lingüístico relacionado con la gramática y el léxico, un saber interaccional relacionado con lo ilocucionario, las máximas y las normas comunicativas y un saber sobre esquemas textuales relacionado con la organización de la información.

Estos conocimientos intuitivos que demuestra el escritor durante la actividad comunicativa deben ser ampliados para una utilización estratégica de los mismos. En especial, la producción escrita requiere para su concreción efectiva que dichos saberes sean profundizados. El uso consciente de estos saberes se hace evidente en las producciones de los escritores competentes, quienes controlan la tarea de elaboración escrita mediante la aplicación de estrategias metacognitivas, es decir, de reflexión sobre su propio hacer. Ello posibilita la regulación de sus propios procesos y productos cognitivos.

Los escritores principiantes deben encontrar en la escuela un espacio donde desarrollen los saberes específicos para producir textos escritos. $\mathrm{Si}$, como ya lo hemos explicitado, las teorías actuales acuerdan en definir la escritura como un proceso complejo que exige la aplicación de estrategias cognitivas por parte del escritor, la posibilidad de discriminar estas estrategias y autorregularlas, implica el desarrollo de estrategias metacognitivas.

Al respecto, a partir de la década de los años 70 ha surgido un gran número de investigaciones 
metacognitivas que tienen que ver con la instrucción y el aprendizaje y en especial, estudian los procesos metacognitivos presentes en la resolución de problemas. En este sentido, distintos teóricos (entre ellos Brown, 1980; Chi \& Glaser, 1986; Martí, 1995; Peronard, 1996) coinciden en señalar que el término "metacognición" fue acuñado por el psicólogo canadiense John Flavell a principios de la década de 1970 en sus investigaciones sobre el desarrollo de los procesos de la memoria, la cognición y la metacognición, definiéndola como el control que las personas realizan de sus propios procesos cognitivos para ser eficientes.

En estos trabajos se hace hincapié en que el estudio de la metacognición puede conducir a identificar importantes mecanismos de cambio y que es indispensable basarse en los resultados de los estudios metacognitivos para diseñar modelos de instrucción, sobre todo para alumnos con dificultades educativas especiales (Martí, 1995).

\subsection{Los estudios metacognitivos y su influencia en los aprendizajes}

En los estudios de Flavell se pone de relieve la importancia del control y la toma de conciencia de los procesos cognitivos por parte del alumno y se buscan las situaciones que las potencien. Su razonamiento se basó en datos empíricos que mostraban que algunos niños podían tener estrategias adecuadas de memoria. Pero no siempre las utilizaban en forma correcta, mostraban "deficiencias de producción". Esto lo llevó a suponer que no es suficiente distinguir el nivel de funcionamiento cognitivo, lo que el niño sabe, sino también cómo controla sus propios procesos cognitivos para ser eficiente en determinadas tareas (nivel de funcionamiento metacognitivo).

Al respecto Flavell (1996: 160) asevera:

"Podríamos decir que se recurre a las estrategias cognitivas para hacer un progreso cognitivo, y a las estrategias metacognitivas para controlarlo. Controlar el propio progreso en una tarea es una actividad metacognitiva muy importante".

En este sentido, Flavell identifica el área metacognitiva como un nuevo campo de investigación que permite entender con mayor precisión cómo los niños van adquiriendo un más amplio conocimiento sobre los procesos cognitivos y cómo los van regulando con mayor eficacia para resolver determinadas tareas. En el modelo que propone este investigador, se identifican dos dominios metacognitivos: el del conocimiento metacognitivo y el de la experiencia metacognitiva. El primero se refiere a los conocimientos que tienen los sujetos sobre la cognición, referido a personas (lo que saben las personas respecto a sus capacidades o a la de otras personas ya sea en lo intraindividual, interindividual o universal), en relación con tareas (el conocimiento sobre demandas y exigencias que conlleva determinada tarea) y en relación con estrategias (conocimiento sobre qué estrategia utilizar para lograr un objetivo y cómo controlar su eficacia o pertinencia según las circunstancias). 
En cuanto al segundo dominio metacognitivo propuesto por Flavell, las experiencias metacognitivas son definidas por el investigador como las sensaciones que experimenta conscientemente un sujeto que está llevando a cabo un proceso cognitivo: darse cuenta del grado de dificultad de la tarea que se está realizando, o tener la sensación de que la vía elegida para resolverla es inadecuada, o sentir que se está muy cerca de alcanzar el objetivo, etc.

Otro avance importante en el área de la metacognición se debe a los estudios de Baker y Brown (Brown, 1980; Baker \& Brown, 1984) quienes trabajan el concepto de metacognición aplicado a la lectura. Asimismo, un trabajo posterior de Brown (1987), constituye, según Martí (1995), una de las contribuciones más sistemáticas al análisis del concepto de metacognición y de las investigaciones relativas al mismo.

Todos estos aportes muestran que aprender haciendo es necesario, pero no suficiente. Los aprendizajes que se están construyendo se ven reforzados y consolidados por una reflexión del propio aprendiz sobre ellos. De esta manera, la metacognición contribuye al desarrollo de los conocimientos tanto declarativos como procedurales. Por su parte, el conocimiento metacognitivo también se va reforzando y acumulando lentamente a lo largo de los años integrándose al "almacén de conocimientos metacognitivos" que cada persona va desarrollando (Flavell, 1996). Según Flavell, parece probable que los conocimientos y las experiencias cognitivas y metacognitivas están constantemente informándose e interactuando en cualquier actividad cognitiva.

Para ello, es preciso que los estudiantes descubran sus propios recursos y regulen conscientemente sus procesos de escritura. Ya que la producción escrita se ha planteado como un proceso de resolución de problemas con distintos condicionamientos, para su solución, se hace necesaria la aplicación de estrategias cognitivas específicas durante la tarea de escritura. Estas estrategias pueden aprenderse mediante actividades que muestren al estudiante la utilidad de las mismas, y que lleven a su conceptualización, es decir, que promuevan la construcción de conocimientos no solo procedurales sino también declarativos o conceptuales. Asimismo, las estrategias metacognitivas pueden ejercitarse, aprenderse y conceptualizarse. Las mismas serán de utilidad, en el campo específico que estamos abordando, para regular, modificar o corregir los procedimientos que entran en juego en la escritura, como también, para evaluar los productos de esta actividad.

\section{2. ¿Es posible plantear una didáctica de la metacognición?}

Antes de iniciar el desarrollo de este apartado nos formulamos algunas interrogantes básicas:

- ¿Se enseña a escribir reflexivamente en la escuela?

- ¿Por qué los alumnos demuestran poca familiarización y poca motivación cuando se les propone escribir y menos aún, cuando se les propone revisar sus propios textos o los de otros compañeros? 
- ¿Por qué, en general, la actividad de revisar textos es una práctica exclusiva del docente, y los alumnos se limitan a recibir de manera pasiva las observaciones del profesor?

- ¿Será posible la ruptura de esta "cultura escolar" para avanzar en el desarrollo de la cognición y de la metacognición?

Después de varios años de aplicación de modelos cognitivos y de las numerosas investigaciones que señalan la estricta relación que hay entre la aplicación de estrategias metacognitivas y el desempeño en las tareas de escritura, creemos que estas interrogantes ya tendrían que estar solucionados. La realidad en las prácticas de producción escrita de nuestro medio evidencia que no es así. En este sentido, coincidimos con Monereo (1994) en que es posible enseñar a los estudiantes a ser conscientes de las decisiones que toman, de las condiciones que deben guiar esas decisiones y de las operaciones que deben poner en marcha para hacerlas efectivas, regulando en todo momento su ajuste y orientación. Pero, para ello, como sostiene este investigador, es fundamental que el profesor sea capaz de modificar sus prácticas educativas de manera tal que introduzca los planteamientos metacognitivos en el aula y que él mismo monitoree sus propios procesos. Es así como expresa:

"...esto solo será factible cuando los profesores seamos conscientes de nuestras propias orientaciones epistemológicas, disciplinarias y didácticas, y tratemos de analizarlas y optimizarlas, con la ayuda de nuestros compañeros (formación continua) y de otros profesionales (asesoramiento)" (Monereo, 1994: 5).

En la actualidad, el papel de mediador que realiza el profesor en clase, cuando trata de "negociar y compartir sus significados" con los alumnos, es admitido y sustentado por distintas teorías. La función mediacional consiste en transferir de forma paulatina el control de cada actividad educativa a los estudiantes.

Esta transferencia de control de una tarea supone una transferencia de la conciencia que tiene el docente sobre cuáles son los mejores procedimientos que pueden utilizarse para aprender un contenido y sobre las condiciones en que está indicada su utilización.

Monereo (1994) sugiere tres principios generales que deberían presidir una didáctica de inspiración metacognitiva.

1. Enseñar a los estudiantes a conocerse mejor como "aprendices". Esto supone ayudarlos a identificar sus dificultades, habilidades y preferencias en el momento de aprender, con una doble finalidad: a) conseguir un mejor ajuste entre sus expectativas de éxito y los resultados obtenidos; y b) facilitar la posibilidad de que adapten las tareas escolares a sus propias características. En síntesis: ayudarles a construir su propia identidad o autoimagen cognitiva.

2. Enseñar a los alumnos a reflexionar sobre su propia manera de aprender, guiarlos para que 
analicen las decisiones regulativas que toman durante la planificación y monitoreen y valoren sus actuaciones cuando realizan una tarea. Es decir, enseñarles a que mejoren la regulación de los procesos cognitivos implicados.

3. Enseñar a los estudiantes a establecer con ellos mismos un diálogo consciente cuando aprenden, o sea, ayudarles a: identificar el propósito de aprendizaje, reconocer las intenciones de quien propone la tarea para ajustarse mejor a las expectativas y demandas y activar sus conocimientos previos sobre los contenidos tratados con el fin de conseguir la elaboración de relaciones sustanciales con la nueva información, logrando un aprendizaje más significativo.

De los aspectos desarrollados en este apartado se desprende una tercera premisa para nuestro modelo:

- La necesidad de la aplicación de estrategias cognitivas y metacognitivas durante la producción.

\section{Modelo didáctico de fases interactivas para la enseñanza y evaluación de la producción escrita (Lacon \& Ortega, 2003)}

A partir de los aportes teóricos mencionados, diseñamos un modelo didáctico de producción escrita en fases interactivas para su puesta en práctica en el aula. Se complementa con los criterios y pautas para su evaluación. La evaluación forma parte del proceso de enseñanzaaprendizaje y está presente de la primera a la última fase. Es necesario destacar, teniendo en cuenta lo señalado en el marco teórico acerca de la importancia de lo motivacional y afectivo en los aprendizajes, que nuestra propuesta está enmarcada en un Proyecto interdisciplinario con un eje temático cercano a los intereses de los estudiantes. El eje del proyecto (utilizado en la concreción de la investigación) "Conozcamos la ciudad de Mendoza" se centra en el propio contexto geográfico y vivencial de la realidad cotidiana del alumno.

Nuestro modelo se estructura en fases. Concebimos cada fase como un conjunto de actividades destinadas a ir desarrollando en forma de complejidad creciente las habilidades de los alumnos para producir textos escritos. Pretendemos con estas propuestas graduales que los alumnos conozcan las estrategias, sean capaces de aplicarlas y lleguen a su utilización eficaz y flexible. Ello supone un progreso cognitivo y un control metacognitivo por parte del alumno y un papel activo del docente para el logro de los resultados esperados. De ahí que, en la aplicación del modelo, sea necesario que el docente, como mediador, apoye a los alumnos en el reconocimiento y conceptualización de estrategias. En este aspecto, es fundamental el uso del intercambio verbal orientado por el profesor, por medio de preguntas destinadas a que los alumnos infieran los nuevos conocimientos y los clarifiquen. Asimismo, es importante la explicitación de los objetivos 
de las tareas a fin de que los estudiantes sepan con claridad cuál es la meta a conseguir y cuál es su utilidad. Ello contribuye a la puesta en marcha de los procesos ejecutivos de comprensión y producción.

Además, como se verá en la explicación del modelo, algunas tareas convienen que sean resueltas en forma colectiva, por el grupo clase. Luego, un trabajo similar puede ser realizado en grupos pequeños conformados por dos alumnos, quienes trabajarán en forma cooperativa. Finalmente, cada alumno podrá enfrentar su tarea de escritura en forma individual.

Deseamos destacar que si bien el modelo contempla la realización de ocho fases, esto no implica que deba ser aplicado tal como está presentado, a modo de "receta"; podrá ser adaptado a las necesidades de cada grupo y a la enseñanza de distintos tipos textuales. El modelo está sustentado en el postulado básico de que la escritura es un proceso complejo, recursivo, flexible e interactivo y como tal debe ser abordado, como así también en las premisas, ya señaladas, que se tienen presentes en las distintas fases.

En función del propósito de este artículo, efectuaremos una presentación sintética de cada una de las fases que se incluyen: objetivos, estrategias y actividades específicas de cada una de ellas y solo nos detendremos -para una mejor clarificación del modelo- a describir con más detalles las fases 3 y 5 .

1. "Diagnóstico de la competencia productiva de los estudiantes". En esta fase los alumnos producen un texto dados los parámetros de una situación comunicativa concreta. Posteriormente, se les solicita que expliquen por escrito cómo procedieron para redactar su texto (informe metacognitivo).

En el trabajo empírico realizado, la consigna de escritura que dimos a los alumnos fue que debían realizar una descripción de la fiesta de la Vendimia de Mendoza $^{2}$ que se publicaría en un folleto turístico de la provincia con distribución en el extranjero.

Para el informe metacognitivo, la consigna fue presentada en forma escrita, pero se dieron precisiones orales sobre la misma para su mejor comprensión. Nuestro interés, en este caso, fue explorar las representaciones internas de los estudiantes sobre el proceso realizado, para su posterior análisis lo que nos permitió comprender mejor la naturaleza de las dificultades halladas.

2. "Procesamiento estratégico de textos expositivos": en esta fase se realiza una actividad preliminar de lectura y comprensión de textos modélicos, de apoyo para el abordaje de la enseñanza-aprendizaje de la producción escrita.

Además del procesamiento estratégico del texto en sus distintos niveles, se analizan los propósitos de las estrategias de lectura llevadas a cabo. Esta actividad metacognitiva apunta a que los 
alumnos reflexionen sobre las estrategias puestas en juego durante la lectura, las interpreten y tomen conciencia de la utilidad de las mismas, a fin de propiciar, paulatinamente, su aplicación automática.

3. "Conocimiento de estrategias de escritura": consiste en el análisis realizado en forma colectiva y guiada por el docente, de uno de los textos leídos. Se trabajan con los alumnos estrategias de producción y de proceso en forma interrelacionada. Se parte de una serie de interrogantes con el objeto de que los alumnos tomen conciencia de las estrategias utilizadas en los distintos niveles del texto y del proceso estratégico seguido por el escritor para su elaboración.

Al finalizar se realiza una recapitulación de lo aprendido. Esto posibilita la elaboración de dos cuadros. Al primero de ellos, que sintetiza las estrategias de producción, lo hemos titulado "Estrategias psicolingüísticas de producción". Al segundo, que esquematiza las estrategias específicas del proceso de escritura, lo denominamos "Estrategias del proceso de escritura". Estos dos cuadros guía, en conjunto, conforman lo que hemos llamado "Estrategias del buen escritor".

El objetivo de la elaboración de estos cuadros, realizados en forma interactiva con los alumnos, es que sean un apoyo, un aporte facilitador o mediación externa que les permita visualizar y recordar todas las estrategias a poner en juego en el momento de escribir un texto.

Pasamos a detallar cómo organizamos esta actividad de enseñanza-aprendizaje de estrategias de escritura:

\section{- Abordaje de estrategias psicolinguiísticas de producción}

En primer lugar se indagan y evalúan las estrategias puestas en juego por el autor en los distintos niveles del texto: pragmático, superestructural, macroestructural, microestructural y proposicional.

A fin de que se advierta el proceso metodológico seguido explicaremos el trabajo realizado para el reconocimiento de las estrategias aplicadas en uno de los niveles, el superestructural.

- Estrategias superestructurales: En interacción con los alumnos se analiza cómo está "presentado" el texto, título destacado, párrafos con sangría inicial, datos bibliográficos y se consideran las distintas funciones clarificadoras que cumplen los elementos paratextuales: el título, anticipar contenidos; el párrafo, delimitar y estructurar la información; la referencia bibliográfica, informar de dónde se ha extraído el texto; las ilustraciones, tal como se desprende de su significado, "mostrar" la función del tipo de texto usado por el autor y en qué lugares suele encontrarse este tipo de texto que proporciona información. Además, se recuerdan cuáles son las categorías canónicas del texto expositivo y se identifican y marcan las que presenta el texto. 
También se determina el porqué de la forma organizacional "descriptiva" y se señalan las marcas lingüísticas propias de la "descripción" -sustantivos, adjetivos y construcciones equivalentes, y verbos presentativos en indicativo- reconociéndose su función en el texto: nombrar los elementos, definirlos o caracterizarlos y presentarlos, respectivamente.

Estas observaciones permiten sistematizar las características del formato descriptivo de los textos expositivos y se elabora la superestructura del mismo.

En síntesis, la reflexión que se realiza con los alumnos en este nivel hace posible que reconozcan cuál es la finalidad comunicativa del emisor y qué tipo de texto es el adecuado para cumplir con ello. Asimismo, qué condicionamientos organizativos y verbales plantea el tipo de texto seleccionado y qué estrategias usa el escritor para responder a estas exigencias.

En este punto, cabe una observación válida para el análisis de las estrategias puestas en juego en otros niveles del texto. Si bien hemos enfocado el nivel superestructural, la necesaria alusión a los otros niveles globales y locales evidencia cómo las estrategias actúan de modo interactivo.

La instancia final de este momento está dada, como hemos señalado más arriba, por la elaboración en forma conjunta del siguiente cuadro guía, sintetizador de las estrategias psicolingüísticas de producción, que servirá de apoyo al alumno para enfrentar las tareas de escritura que se proponen en las fases siguientes. 
Cuadro 1. Cuadro guía: Estrategias psicolingüísticas de producción.

\begin{tabular}{|c|c|c|}
\hline Niveles & Estrategias & Explicitación de las estrategias \\
\hline Pragmático & $\begin{array}{l}\text { Adecuación a la situación } \\
\text { comunicativa }\end{array}$ & $\begin{array}{l}\text { Ajuste a las características del receptor, al contexto } \\
\text { comunicativo y al propósito del escrito: informar } \\
\text { objetivamente, lengua estándar, registro general, } \\
\text { escrito y formal }\end{array}$ \\
\hline Superestructural & $\begin{array}{l}\text { Ajuste a las categorías } \\
\text { canónicas } \\
\text { Ajuste a las exigencias } \\
\text { de las categorías } \\
\text { procedimentales incluidas } \\
\text { Adecuada diagramación }\end{array}$ & $\begin{array}{l}\text { Introducción y desarrollo, conclusión (optativa) } \\
\text { Adecuados procedimientos para el desarrollo } \\
\text { temático. } \\
\text { Párrafos con sangría inicial }\end{array}$ \\
\hline Macroestructural & $\begin{array}{l}\text { Mantenimiento de unidad } \\
\text { temática } \\
\text { Cohesión entre } \\
\text { macroproposiciones }\end{array}$ & $\begin{array}{l}\text { Tema global del primer párrafo, eje unificador de } \\
\text { temas parciales de los otros párrafos. } \\
\text { Conexión entre los párrafos léxica y/o gramatical. } \\
\text { Puntuación delimitadora: punto y aparte }\end{array}$ \\
\hline Microestructural & $\begin{array}{l}\text { Adecuada progresión } \\
\text { temática } \\
\text { Cohesión interoracional }\end{array}$ & $\begin{array}{l}\text { Progresión ordenada por temas enunciados en posición } \\
\text { inicial y remas que los especifican y/o explican. } \\
\text { Conexión léxica o gramatical entre oraciones. } \\
\text { Adecuada selección de sustitutos. Puntuación } \\
\text { delimitadora: punto seguido }\end{array}$ \\
\hline Proposicional & $\begin{array}{l}\text { Correcta estructuración } \\
\text { sintáctica } \\
\text { Adecuada relación } \\
\text { semántica }\end{array}$ & $\begin{array}{l}\text { Concordancia de género y número; correlación verbal } \\
\text { entre períodos sintácticos. Adecuadas conexiones de } \\
\text { coordinación y subordinación } \\
\text { Ordenada organización de constituyentes oracionales } \\
\text { Adecuada elección del léxico y de sus sustitutos. } \\
\text { Información pertinente, no errónea, no repetitiva, } \\
\text { sin contradicciones }\end{array}$ \\
\hline
\end{tabular}

\section{- Estrategias del proceso de escritura}

Para enfocar el proceso de producción seguimos, básicamente, los modelos teóricos de Flower y Hayes $(1981,1996)$ y de Hayes (1996). La elucidación de las estrategias cognitivas representadas en estos modelos en conjunción con las señaladas en el modelo estratégico de van Dijk y Kinstch (1983), posibilita ubicar a los alumnos, en el complejo problema de elaborar textos escritos.

Procedemos a explicar el trabajo realizado:

A partir de una serie de interrogantes que se plantea a los alumnos, se trata de que tomen conciencia de las distintas fases del proceso de escritura y de las estrategias a aplicar en cada una de ellas. 
Las preguntas dan lugar al intercambio verbal o discusión del grupo clase. El docente debe procurar que esta interacción sea un medio relevante para que se enriquezcan las observaciones, se explicite cada fase del proceso de escritura y se reconozcan los procedimientos estratégicos puestos en juego por el autor en su tarea. La serie de interrogantes pretende ser una pista que active la observación y el análisis de los distintos niveles de operaciones implicados en el proceso de redacción.

Las respuestas y los comentarios a que dieron lugar permitieron elaborar el siguiente cuadro:

Cuadro 2. Cuadro guía: Estrategias del proceso de escritura.

(operaciones que pueden repetirse, recursivas):

Estrategias de planificación:

- determinación de la situación retórica:

- determinar qué se quiere decir (tema).

- pensar para qué se escribe (objetivos del escritor).

- tener en cuenta para quién se escribe (audiencia) y la posición de sí mismo como emisor (en calidad de qué se escribe).

- generación de ideas:

- pensar qué ideas se desarrollarán.

- observar, tomar notas, consultar bibliografía, etc., según el tipo de texto y el propósito del escrito.

- organización de las ideas:

- dar forma a las ideas en un esquema, listado, cuadro sinóptico, etc., teniendo en cuenta las ideas generadas y la superestructura del texto a producir (objetivos de escritura).

Estrategias de escritura:

- escritura en borrador:

- redactar un borrador apoyándose en lo planificado y en los objetivos de escritura planteados previamente: situación retórica, generación y organización de ideas.

- revisión, corrección, reescritura:

- revisar lo escrito, evaluarlo en sus distintos niveles, corregir, planificar, reescribir. 
4. "Evaluación colectiva de un escrito producido por un alumno y corrección estratégica": esta fase está centrada en la reflexión sobre el propio proceso de escritura y sobre las estrategias de producción. Para ello se analiza en forma conjunta uno de los textos escritos por los alumnos en el pretest. Los dos cuadros guía, que en conjunto conforman las "Estrategias del buen escritor", sirven como apoyo de la tarea.

Se focalizan los problemas encontrados en los distintos niveles y se proponen las correcciones necesarias. Posteriormente, cada alumno revisa y reescribe su propio texto. El nuevo producto obtenido es revisado, nuevamente, por dos compañeros antes de ser entregado al profesor.

5. "Aplicación y reconocimiento de estrategias en ejercicios de escritura": plantea ejercicios de escritura breves y graduados, a modo de situaciones problemáticas, que el alumno debe resolver usando estrategias de escritura. Asimismo, el estudiante debe explicitar cuáles son las estrategias -de proceso y de producto- aplicadas en cada ocasión. Los ejercicios son revisados entre pares y corregidos en forma colectiva. De esta manera, se logra que los alumnos vayan afianzando, en forma paulatina, su competencia productiva.

Para una mejor comprensión, transcribimos a continuación dos de los ejercicios realizados con los alumnos en esta fase.

Práctica de observación, enumeración, selección y escritura (esta práctica se realizó a partir de la observación de un elemento real llevado al aula: una planta).

a. Observa la planta que te presentamos y piensa cómo la describirías. Escribe una lista de todas sus partes y sus características. Luego organiza los datos en un esquema que refleje el orden que le darías en un texto expositivo-descriptivo.

b. Completa el ejercicio mencionando qué estrategias aplicaste para resolverlo.

Estrategias aplicadas:

Práctica de adaptación al lector, inserción y reescritura.

Reescribe el siguiente texto insertando definiciones, ejemplos y/o reformulaciones que permitan una mayor comprensión del mismo por parte de una persona no especializada en el tema.

Completa el ejercicio mencionando qué estrategias aplicaste para resolverlo.

"Para clasificar los árboles de acuerdo con un valor de finalidad se distinguen tres categorías: árboles "forestales", que son los que constituyen los bosques y se cultivan únicamente por el valor de su madera; árboles "frutales", entre los que figuran los cultivados por los frutos comestibles que proporcionan y, en fin, árboles "ornamentales", que son los que ofrecen un interés estético individual o de masa" (Monitor. Buenos Aires: Salvat, 1966, p. 422).

Estrategias aplicadas: 
"Producción de textos escritos" enfrenta a los estudiantes al desafío de escribir un texto adecuado a una situación comunicativa concreta, poniendo en juego las estrategias adquiridas y desarrolladas en este período de instrucción. Las tareas de revisión y reflexión sobre la aplicación de estrategias son apoyadas por el docente y, en forma colaborativa, por otros alumnos.

La última actividad de esta fase, con el propósito de consolidar la reflexión metacognitiva sobre el proceso de escritura que se ha seguido, consiste en la elaboración de un informe metacognitivo. Se solicita a distintos alumnos que lo expliciten en forma oral. El resto de la clase también participa, no solo como escucha, sino reafirmando, colaborando o haciendo acotaciones.

6. "Producción final o postest e informe metacognitivo de los estudiantes": propone que los alumnos realicen en forma individual un último escrito, el postest.

Al igual que se hiciera luego del pretest, se procede a solicitar a los estudiantes, una vez terminada su redacción, que expliquen, por escrito, de qué manera han procedido para producir su texto. Con esta tarea se pretende explorar las representaciones de los alumnos con respecto a la tarea de escritura.

Las condiciones de realización del postest son similares a las del pretest. Antes de escribir se dialoga acerca del propósito de escritura, el tema, el potencial lector y las circunstancias de producción. En la instancia experimental se acordó la escritura de un texto expositivo-descriptivo sobre un lugar previamente visitado por los alumnos: la Plaza Independencia ${ }^{3}$. Una vez acordados los parámetros de la situación de producción se trabaja en forma individual.

7. "Reflexión y análisis de resultados": profesor y alumnos cotejan los resultados del pretest y postest y los informes metacognitivos escritos por los alumnos en ambas oportunidades. Ello da pie a una sesión colectiva de discusión, análisis y formulación de conclusiones.

El Cuadro 3 permite apreciar en forma global el desenvolvimiento de las fases descriptas.

Cuadro 3. Modelo didáctico de fases interactivas de enseñanza de la producción.

\begin{tabular}{|c|c|c|}
\hline Objetivos & Estrategias & Tareas globales \\
\hline $\begin{array}{l}\text { 1. Escribir un texto dentro de } \\
\text { los parámetros de una situación } \\
\text { comunicativa consensuada }\end{array}$ & $\begin{array}{l}\text { De producción } \\
\text { Metacognitivas }\end{array}$ & $\begin{array}{l}\text { Escritura inicial de un texto o pretest y de la } \\
\text { explicación del proceso seguido para escribir- } \\
\text { informe metacognitivo. }\end{array}$ \\
\hline
\end{tabular}




\begin{tabular}{|c|c|c|}
\hline \multirow{4}{*}{$\begin{array}{l}\text { 2. Construir una representación } \\
\text { del texto en la memoria } \\
\text { aplicando estrategias de } \\
\text { lectura }\end{array}$} & De prelectura & $\begin{array}{l}\text { Formulación de hipótesis a partir de las marcas } \\
\text { paratextuales de un texto. }\end{array}$ \\
\hline & De lectura & $\begin{array}{l}\text { Procesamiento estratégico del texto en sus distintos } \\
\text { niveles. }\end{array}$ \\
\hline & De poslectura & Organización gráfica del contenido del texto. \\
\hline & Metacognitivas & $\begin{array}{l}\text { Reflexión sobre los propósitos de las estrategias de } \\
\text { lectura llevadas a cabo. Elaboración de conclusiones } \\
\text { en forma oral y escrita. }\end{array}$ \\
\hline \multirow[t]{2}{*}{$\begin{array}{l}\text { 3. Construir un modelo } \\
\text { adecuado de producción escrita } \\
\text { en la memoria }\end{array}$} & $\begin{array}{l}\text { De producción } \\
\text { psicolingüísticas } \\
\text { Cognitivas y } \\
\text { metacognitivas }\end{array}$ & $\begin{array}{l}\text { Evaluación del texto leído y reconocimiento de las } \\
\text { estrategias de escritura usadas por el autor en sus } \\
\text { distintos niveles. } \\
\text { Elaboración de un cuadro síntesis de las estrategias } \\
\text { psicolingüísticas de producción. }\end{array}$ \\
\hline & $\begin{array}{l}\text { Del proceso } \\
\text { de escritura, } \\
\text { cognitivas y } \\
\text { metacognitivas }\end{array}$ & $\begin{array}{l}\text { Comprensión y reconocimiento de las fases del } \\
\text { proceso de escritura. } \\
\text { Reflexión, explicitación oral y síntesis escrita sobre } \\
\text { las estrategias puestas en juego en el proceso de } \\
\text { escritura. }\end{array}$ \\
\hline $\begin{array}{l}\text { 4.Proponer estrategias } \\
\text { correctoras de los escritos }\end{array}$ & $\begin{array}{l}\text { De lectura } \\
\text { y escritura } \\
\text { interconectadas } \\
\text { Metacognitivas }\end{array}$ & $\begin{array}{l}\text { Evaluación colectiva de un escrito. } \\
\text { Evaluación, corrección cooperativa y luego } \\
\text { individual del texto personal elaborado }\end{array}$ \\
\hline $\begin{array}{l}\text { 5. Aplicar estrategias de } \\
\text { escritura }\end{array}$ & $\begin{array}{l}\text { Estrategias de } \\
\text { lectura y escritura } \\
\text { interconectadas } \\
\text { Metacognitivas }\end{array}$ & $\begin{array}{l}\text { Aplicación de estrategias en ejercicios de escritura } \\
\text { breves y graduados } \\
\text { Identificación de las estrategias puestas en juego } \\
\text { en cada actividad }\end{array}$ \\
\hline $\begin{array}{l}\text { 6. Producir textos aplicando } \\
\text { reflexivamente estrategias de } \\
\text { escritura, con apoyo externo } \\
\text { en las tareas de revisión y } \\
\text { corrección }\end{array}$ & Metacognitivas & $\begin{array}{l}\text { Producción de textos poniendo en marcha las } \\
\text { estrategias aprendidas para escribir, corregir y } \\
\text { reescribir } \\
\text { Revisión y regulación de la tarea en forma } \\
\text { colaborativa. }\end{array}$ \\
\hline $\begin{array}{l}\text { 7. Escribir un } \\
\text { texto y monitorear } \\
\text { autorreflexivamente el texto } \\
\text { que se va produciendo y el } \\
\text { producto final. } \\
\text { Determinar reflexivamente las } \\
\text { estrategias aplicadas }\end{array}$ & $\begin{array}{l}\text { De escritura } \\
\text { Metacognitivas }\end{array}$ & $\begin{array}{l}\text { Producción final o postest. } \\
\text { Elaboración de un informe metacognitivo sobre } \\
\text { las estrategias puestas en juego en la producción } \\
\text { personal. }\end{array}$ \\
\hline $\begin{array}{l}\text { 8. Reflexionar sobre el proceso } \\
\text { de aprendizaje seguido y } \\
\text { elaborar conclusiones }\end{array}$ & $\begin{array}{l}\text { Cognitivas y } \\
\text { metacognitivas }\end{array}$ & $\begin{array}{l}\text { Sesión colectiva de discusión, análisis y } \\
\text { formulación de conclusiones. }\end{array}$ \\
\hline
\end{tabular}


A efectos de que el lector pueda apreciar la evolución seguida durante el proceso de aplicación del modelo de fases interactivas, del pretest al postest, transcribimos, en el Anexo, las producciones de un alumno, en su versión original y los informes metacognitivos realizados en estas dos instancias.

\section{CONCLUSIONES}

A la luz de lo expuesto podemos concluir que:

- Nuestra propuesta metodológica ofrece una alternativa de solución a la problemática de la escritura tanto en nivel básico, medio o polimodal como en el universitario.

- En nuestro modelo de enseñanza hemos logrado integrar modelos de producción escrita flexibles y funcionales que se complementan entre sí, apoyándonos, también, en teorías metacognitivas.

- En todos los casos, será necesario que las tareas de escritura estén encuadradas en una situación comunicativa concreta y tengan un propósito determinado, a fin de que el alumno sea consciente de la pertinencia e importancia de emitir su mensaje en un contexto sociocultural específico. Ello puede darse, con mayor eficacia, si encuadramos la enseñanza de la escritura en un proyecto interdisciplinario.

- No es conveniente ser rígido en la enseñanza de las estrategias, pero sí desarrollar la tarea de enseñanza-aprendizaje-evaluación en forma sistemática e intensiva.

- La práctica de las estrategias debe tener un carácter gradual. En otras palabras, creemos conveniente disponer la situación de aprendizaje de modo que la estrategia se practique en primer lugar sobre tareas más sencillas, para posteriormente evolucionar hacia tareas más complejas, de manera de proporcionar al alumno una serie de experiencias de éxito en la aplicación de la estrategia que pueden suponer un factor crucial en el desarrollo de su competencia productiva.

- Los alumnos deben lograr niveles de conciencia cada vez más elevados a lo largo de todo el proceso de producción. Debido a ello se enfatizan en todas las fases las tareas de índole metacognitiva tal como hemos explicitado. Con esto se busca cambiar la concepción que los alumnos tienen de la escritura, que conozcan y se involucren en los procesos que realizan al escribir de manera tal que, progresivamente, sean capaces de construir su propia identidad como escritores, logren regular los procesos cognitivos implicados, puedan activar sus conocimientos previos y elaborar relaciones sustanciales con la nueva información para lograr un aprendizaje significativo. Estos logros permitirían afirmar que sí es posible aplicar una didáctica de la metacognición a fin de que los escritores principiantes sean capaces de poner en marcha procesos de producción cada vez más complejos. 
- El éxito en la aplicación de este o de cualquier modelo está en estricta relación con el carácter de las intervenciones didácticas y, fundamentalmente, con el logro de un entendimiento interpersonal profesor/alumno, en la relación dialógica.

\section{REFERENCIAS BIBLIOGRÁFICAS}

Bajtín, M. (1982). Estética de la creación verbal. México, D.F.: Siglo XXI.

Baker, L. \& Brown, A. (1984). Metacognitive skill and reading. En D. Pearson, R. Barr, M. Kamil \& P. Mosenthal (Comps.), Handbook of reading research (pp. 353-394). New York: Longmann.

Badura, B. (1972). Kommunikative kompetenz, Dialog, Hermeneutik und Interaction: Eine theoretische Skizze. En B. Badura \& K. Gloy (Eds.), Soziologie der Kommunikation. Eine Textauswahl zur Einführung (pp. 246-264). Stuttgart: Frommann-Holzboog.

Bereiter, C. \& Scardamalia, M. (1987). The psichology of writen composition. Hillsdale, N.J.: Erlbaum.

Brown, A. (1980). Metacognitive development and reading. En R. Spiro, B. Bruce \& W. Brewer (Eds.), Theorical issues in reading comprehension (pp. 453-481). Hillsdale, N.J.: Erlbaum.

Brown, A. (1987). Metacognition, executive control, self-regulation and other more mysterious mechanisms. En F. Weinert \& H. Kluwe (Eds.), Learning by thinking (pp. 65-116). Hillsdale, N.J.: Erlbaum.

Camps, A. (1993). La enseñanza de la composición escrita. Cuadernos de Pedagogía, 216, 19-21.

Camps, A. (1997). Escribir. La enseñanza y el aprendizaje de la composición escrita. Signos. Teoría y prácticas de la educación, 20, 24-33.

Camps, A. \& Castelló, M. (1996). Las estrategias de enseñanza-aprendizaje en la escritura. En C. Monereo \& I. Solé (Coords.), El asesoramiento psicopedagógico: Una perspectiva profesional y constructivista (pp. 321-342). Madrid: Alianza.

Castelló, M. (2002). De la investigación sobre el proceso de composición a la enseñanza de la escritura. Revista Signos, 52-53, 149-162.

Chi, M. \& Glaser, R. (1986). Las capacidades humanas. Barcelona: Labor.

Dolz, J. (1994). Seqüencies didactiques i enseyament de la llengua: Més enlla dels projectes de lectura i d' escriptura. Articles, 2, 21-34.

Flavell, J. (1996). El desarrollo cognitivo. Madrid: Aprendizaje Visor.

Flower, L. \& Hayes, J. (1980). The cognition of discovery: Defining a rhetorical problem. College composition and Comunication, 31, 21-32.

Flower, L. \& Hayes, J. (1981). A cognitive process theory of writing. College composition and Comunication, 32(4), 365-387.

Flower, L. \& Hayes, J. (1996). La teoría de la redacción como proceso cognitivo. Textos en contexto. Buenos Aires: Asociación Internacional de Lectura. 
Gagné, E. (1991). La psicología cognitiva del aprendizaje escolar. Madrid: Aprendizaje Visor.

Gómez de Erice, M. (2003). Competencia y modelos de comprensión lectora. En M. Gómez de Erice \& E. Zalba (Eds.), Comprensión de textos. Un modelo conceptual y procedimental (pp. 23-33). Mendoza: EDIUNC.

Hayes, J. (1996). Un nuevo marco para la comprensión de lo cognitivo y lo emocional en la escritura. En M. Levy \& S. Ransdell (Eds.), The science of writing (pp. 1-27). New Jersey: Erlbaum.

Hymes, D. (1962). The ethnography of speaking. En T. Gladwin \& W. Sturtevant, (Eds.), Antropology and human behavior (pp. 13-53) Washington, D.C.: The Anthropology Society of Washington.

Heinemann, W. \& Viehweger, D. (1991). Textlinguistik. Eine Einführung. Tübingen: Nemeyer.

Lacon de De Lucia, N. (2001). Estrategias para la producción escrita de textos expositivos. Tesis de Magíster, Universidad Nacional de Cuyo, Mendoza, Argentina.

Lacon de De Lucia, N. \& Ortega de Hocevar, S. (2003). Producción de textos escritos. Mendoza: EDIUNC.

Martí, E. (1995). Metacognición: Entre la fascinación y el desencanto. Revista Infancia y Aprendizaje, 72, 9-32.

Monereo, C. (1994). Enseñar a conciencia (en línea). Disponible en http:// seneca.uab.es/monereo/

Ortega de Hocevar, S. (2000). Estudio sincrónico de producciones infantiles en el primer ciclo de la Educación General Básica, desde el punto de vista léxico-semántico. Tesis de Magíster, Universidad Nacional de Cuyo, Mendoza, Argentina.

Ortega de Hocevar, S. (2005). Proyecto de investigación: La competencia discursiva y metadiscursiva de alumnos que finalizan el primer ciclo de Educación Básica Común y/o su equivalente en educación especial (2002-2005). Mendoza: SECyTP.

Parodi, G. (2003). Relaciones entre lectura y escritura: Una perspectiva cognitiva- discursiva. Valparaíso: EUVSA.

Peronard, M. (1996). Experiencia y conocimiento metacognitivo. Revista Signos, 38-39, 121-131.

Salvador Mata, F. (1997). Dificultades en el aprendizaje de la expresión escrita. Málaga: Aljibe.

van Dijk, T. \& Kintsch, W. (1983). Strategies of discourse comprehension. New York: Academic Press.

Vygotsky, L. (1979). El desarrollo de los procesos psicológicos superiores. Barcelona, Grijalbo.

\section{NOTAS}

1 El presente trabajo sintetiza los principales aspectos de dos investigaciones sobre la producción de textos escritos que llevamos a cabo en forma individual pero paralelas en el tiempo, como sustento de nuestras respectivas Tesis de maestría (Ortega de Hocevar, S., 2000; Lacon de De Lucia, N., 2001), como así también de un Proyecto de Investigación (dirigido por Susana Ortega de Hocevar, 2005). Los resultados de estas investigaciones tuvieron la oportunidad de confrontarse 
y de profundizarse a través de un trabajo que realizamos en conjunto, a raíz de que en el año 2003 fuimos convocadas por la Secretaría Académica de Rectorado, en carácter de consultoras expertas, a efectos de diseñar, escribir y dictar un Módulo destinado a la producción de textos escritos.

2 La Fiesta de la Vendimia es la más representativa de la provincia de Mendoza, Argentina. Está incluida en los calendarios nacionales e internacionales.

3 La Plaza Independencia es la plaza mayor de la ciudad Capital de Mendoza, Argentina. En ella se realizan eventos patrióticos, culturales y artísticos en fechas significativas.

\section{ANEXO}

1. Ejemplos de producciones de los alumnos en las distintas fases de aplicación del modelo.

- Textos escritos en el pretest (sujeto 20 de la muestra)

\section{Borrador realizado en el pretest}

Tema: La fiesta de la vendimia mendocina

Partisipan muchas chicas que quieren ser la reina del departamento donde viven. De las cuales solo gana una la más linda y hay una segunda que es la Virreina. En total son 18 reinas.

En la bendición de los frutos van llevados en un carro llamado Vines tirado por un tractor. Luego lo vendice el Obispo.

En la Vía Blanca, desfilan en un carro todo iluminado arrojando uvas, publicidad y regalan vinos. El carrusel se larga de los portones del parque General San Martín que también desfilan las reinas pero esta ves acompañados de gauchos, caballos y muchas cosas más.

La gran fiesta es un espectacular acto donde hay vaile y canto antes de elejir a la reina nacional de la vendimia. Y a lo último por elección de votos ganan dos la reina y la virreina entre las 18 aspirante.

\section{Producción final del pretest}

\section{La fiesta de la vendimia mendocina}

Participan muchas chicas que quieren ser la reina del departamento donde viven. De las cuales solo gana una la más linda y hay una segunda que es la Virreina. En total son 18 reinas.

En la bendición de los frutos van llevados en un carro llamado Vines tirado por un tractor. Luego los bendice el Obispo.

En la Vía Blanca desfilan en un carro todo iluminado arrojando uvas, publicidad y regalan vinos. El carrusel se larga de los portones del parque General San Martín que también desfilan las reinas pero esta vez acompañadas de gauchos, caballos y muchas cosas más.

La gran fiesta es un espectacular acto donde hay baile y canto antes de elegir a la Reina Nacional de la Vendimia. Y a lo último por elección de votos ganan dos la reina y la virreina entre las 18 aspirantes. 


\section{- Textos escritos en el postest}

\section{Notas previas realizadas por el alumno}

Tema: La plaza Independencia.

La plaza abarca 4 manzanas: entre P. Mendocinas, Chile, Espejo y Rivadavia-Ingreso por peatonal Sarmiento: fuente mediana rectangular, a ambos costados, bancos, caminos, espacios verdes y árboles con nombres científicos. Se hubican artesanos. -Centro de la Plaza: fuente grande semicircular, aguas dansantes, de noche con juego de luces -Arriba fuente y hacia atrás: escenario; debajo y hacia la derecha teatro Quintanilla; hacia la isquierda Museo de Arte Moderno. -Más adelante cerca calle Espejo, juegos para niños -Por Chile en el centro escudo de Mendoza, a la isquierda fuente pequeña y glorieta. -Gran cantidad de faroles en la noche mucha iluminación.

\section{Borrador 1}

La plaza Independencia Por la entrada a la plaza que es por el Oeste por la peatonal, calle Sarmiento se ve una fuente mediana, a ambos costados hay bancos, caminos, a la isquierda hay un espasio verde con árboles que tienen su nombre científico y el comun. Por ahí se hubican artesanos. En el centro de la Plaza hay una fuente grande con aguas danzantes y de noche con juego de luces. Arriba y hacia atrás hay un escenario; debajo y hacia la derecha el teatro Quintanilla y hacia la isquierda el Museo de Arte Moderno. Más adelante, cerca de la calle Espejo, hay juegos para niños.-Por Chile, en el centro, está el escudo de Mendoza. A la isquierda hay una fuente pequeña y una glorieta. Hay gran cantidad de faroles en la noche, dan mucha iluminación.

\section{Borrador 2}

La plaza Independencia.

Para ingresar a la plaza lo podés hacer por distintas calles, por el este, la Peatonal, por el norte, la calle Mitre, por el oeste, la calle Rivadavia, por el norte, la calle Chile.Entrando por la peatonal se ve una fuente mediana a la isquierda hay un espasio verde con árboles que tienen su nombre científico y el comun. En este sector hay puestos de artesanosMás adelante te encontrás la fuente principal, la más destacada, llena de luces en la noche, su forma es semicircular, con aguas dansantes. Arriba y hacia atrás se encuentra un pequeño escenario, abajo del mismo hacia la isquierda está el teatro Quintanilla y hacia la derecha se encuentra el Museo de Arte Moderno.

Subiendo unas escaleras está la glorieta donde se encuentra otra de las fuentes que no tiene mucho agua, en esa parte de la plaza hay mucho verde y distinguidas flores. Hacia atrás y bajando está los juegos para los niños, en esa parte el piso no está hecho de cemento sino es arena muy suave. Hay distintos tipos de juegos como por ejemplo los columpios, toboganes, etc.Desde los juegos y demás lugares de la plaza se puede obserbar y apresiar el gran Escudo Provincial que en la noche es una de las atracciones más emocionantes de la plaza, gracias a su distinguida iluminación.Por toda la plaza hay muchos faroles que la iluminan de noche. 


\section{Texto final}

La Plaza Independencia

La plaza Independencia está ubicada en la zona céntrica de la Ciudad de Mendoza. Es su plaza más importante y es la más concurrida. La superficie de la misma es de cuatro hectáreas, abarca cuatro manzanas. La limitan la calle Rivadavia, al sur, Patricias, al este, Espejo, al norte y Chile, al oeste. Si se entra por la calle Patricias se ve una fuente mediana de forma rectangular. En esa parte siempre hay gente caminando o sentada, descansando en los bancos ubicados a ambos lados de los caminos que bordean la fuente. En las veredas laterales de esta zona se ubican artesanos con sus mercaderías. Más adelante se encuentra la fuente principal, su forma es semicircular. Los chorros de agua que brotan de la misma alcanzan distintas alturas por lo que parecen danzar. De noche, la fuente queda muy destacada por su gran juego de luces. En este lugar se concentra mucha gente principalmente estudiantes ya que hay colegios frente a la plaza.

Arriba y hacia atrás de esta fuente se encuentra un pequeño escenario donde se realizan distintos eventos. Abajo del mismo hacia el sur se ubica un teatro, el Quintanilla y hacia al norte, el Museo de Arte Moderno. Subiendo unas escaleras hay una glorieta y otra fuente más pequeña. Esta parte de la plaza tiene mucho verde y flores de muchos colores. Hacia atrás y bajando las escaleras están los juegos para los niños. En este sector el piso es de arena muy suave. En el sector oeste de la plaza, el que da a la calle Chile, se ubica el gran Escudo Provincial que de noche por su muy buena iluminación se aprecia desde lugares alejados. Por toda la plaza hay espacios verdes con flores, árboles que muestran su nombre científico y el común y muchos faroles que la iluminan de noche. Como la plaza Independencia es la más importante, allí se realiza la elección de la reina de la Capital provincial.

- Informes metacognitivos realizados por el alumno.

\section{Del pretest}

Los pasos que he tenido en cuenta son: pensar cómo son las distintas actividades de la fiesta, luego escribí en una hoja borrador todos las actividades que tiene y la descripción de cada una, me fijé si tenía errores en los signos de puntuación, palabras mal escritas y por último, lo pasé en limpio y listo a una hoja nueva.

\section{Del postest}

Cuando se mencionó el tema pensé qué podría escribir.

En la plaza observé con todos los sentidos y tomé notas. Luego, miré mis notas y las ordené. Recién después analicé, planifiqué lo que iba a escribir, a quién iba dirigido, para qué lo hacía y elegí qué clase de texto hacer.

Revisé el cuadro de estrategias y los esquemas hechos en clase para el tipo de texto expositivo descriptivo. Luego comencé a escribir en orden un borrador, lo revisó un compañero, comentamos $\mathrm{y}$ vimos algunos detalles

Iba escribiendo, borré y volvi a escribir hasta conseguir lo que deseaba. Le pregunté al profe y me hizo ver algunos detalles que corregí. Recordé al corregir lo aprendido en clases y verifiqué con el cuadro de estrategias. Hice cambios en el vocabulario y a quien iba dirigido.

Cuando terminé el texto lo leí, lo volví a revisar y lo pasé en limpio. 